ANNALES

POLONICI MATHEMATICI

$97.3(2010)$

\title{
A stochastic model of symbiosis
}

\author{
by Urszula Skwara (Lublin)
}

\begin{abstract}
We consider a system of stochastic differential equations which models the dynamics of two populations living in symbiosis. We prove the existence, uniqueness and positivity of solutions. We analyse the long-time behaviour of both trajectories and distributions of solutions. We give a biological interpretation of the model.
\end{abstract}

1. Introduction. Relations between two populations living in symbiosis can be described by the following system of differential equations [3] (Gause and Witt, 1935)

$$
x^{\prime}=\left(a_{1}+b_{1} y-c_{1} x\right) x, \quad y^{\prime}=\left(a_{2}+b_{2} x-c_{2} y\right) y .
$$

This model does not take into account the random influence of the environment and the following stochastic model would be more realistic:

$$
\begin{aligned}
& d X(t)=\left(\left(a_{1}+b_{1} Y(t)-c_{1} X(t)\right) d t+\rho_{11} d W_{1}(t)+\rho_{12} d W_{2}(t)\right) X(t), \\
& d Y(t)=\left(\left(a_{2}+b_{2} X(t)-c_{2} Y(t)\right) d t+\rho_{21} d W_{1}(t)+\rho_{22} d W_{2}(t)\right) Y(t),
\end{aligned}
$$

where $a_{i}, b_{i}, c_{i}(i=1,2)$ are positive constants, and $W_{1}(t), W_{2}(t)$ are two independent standard Wiener processes. The stochastic processes $X(t)$ and $Y(t)$ represent, respectively, the first and the second population. The constants $a_{i}(i=1,2)$ are ideal growth rates, $b_{i}(i=1,2)$ are symbiosis coefficients, $c_{i}(i=1,2)$ are death rates and $\rho_{i j}(i, j=1,2)$ are the coefficients of environmental stochastic perturbations of populations.

The classical model of symbiosis described by (1) is well known (see [1], [9]). If the coefficients of system (1) are positive and satisfy the inequality $b_{1} b_{2}<c_{1} c_{2}$ then the sizes of both populations converge to a unique equilibrium. Otherwise, if $b_{1} b_{2} \geq c_{1} c_{2}$ then the sizes go to infinity. In the literature we can find other models of symbiosis. V. A. Kostitzin [6] considered a more complicated model of symbiosis in which, besides free-living individuals in two associated species, we have the third population of symbiotic couples.

2010 Mathematics Subject Classification: 47D07, 35K15, 60J60, 60H10, 92D25.

Key words and phrases: symbiosis model, diffusion process, Markov semigroups, asymptotic stability. 
In [14] A. Rescigno and W. Richardson considered a more general model of symbiosis than (1). They replaced the growth rates $a_{1}+b_{1} Y(t)-c_{1} X(t)$, $a_{2}+b_{2} X(t)-c_{2} Y(t)$ by functions $f_{1}, f_{2}$ which satisfy specific conditions. We can also study stochastic versions of these models using similar methods.

The aim of this paper is to study the long-time behaviour of both trajectories and distributions of the solutions of system (2), (3). First we show the existence, uniqueness, positivity and non-extinction property of the solutions. Next we prove that the probability distributions of the process $(X(t), Y(t))$ are absolutely continuous with respect to the Lebesgue measure. Let $U(x, y, t)$ be the density of the distribution of $(X(t), Y(t))$. We give a sufficient and a necessary condition for the asymptotic stability of system (2), (3), i.e. the convergence of $U(x, y, t)$ to an invariant density $U_{*}(x, y)$. If this system is not asymptotically stable then we prove that $\lim _{t \rightarrow \infty} Y(t)=0$ a.e. We also show that in this case $\lim _{t \rightarrow \infty} X(t)=0$ a.e. or the probability distributions of the process $X(t)$ converge weakly to some probability measure.

In order to prove asymptotic stability of system (2), (3) we use the theory of integral Markov semigroups developed in [11, [12], [15] and [18. If this system is not asymptotically stable then we use the comparison theorem for one-dimensional stochastic differential equations and the ergodic theorem to show that $\lim _{t \rightarrow \infty} Y(t)=0$ a.e. A similar technique was applied to study the asymptotic behaviour of a stochastic prey-predator model [16], [17], a stochastic competition model [20] and a stochastic SIR model [19].

In the model described by equations (2), (3) the random noise is proportional to the number of individuals in the population. This occurs when the noise is caused, for example, by an epidemic disease or weather conditions. We can consider another model of symbiosis in which the random noise affects each individual separately. In this case we assume that the mean value of the stochastic perturbation is zero. From the central limit theorem it follows that the stochastic perturbation is gaussian and proportional to $\sqrt{X(t)}$ and $\sqrt{Y(t)}$ respectively. Then we obtain the system

$$
\begin{aligned}
d X(t)= & \left(a_{1}+b_{1} Y(t)-c_{1} X(t)\right) X(t) d t \\
& +\left(\rho_{11} d W_{1}(t)+\rho_{12} d W_{2}(t)\right) \sqrt{X(t)}, \\
d Y(t)= & \left(\left(a_{2}+b_{2} X(t)-c_{2} Y(t)\right) Y(t) d t+\left(\rho_{21} d W_{1}(t)\right.\right. \\
& \left.+\rho_{22} d W_{2}(t)\right) \sqrt{Y(t)} .
\end{aligned}
$$

The existence, uniqueness, positivity and non-extinction property of the solutions of system (4), (5) follow from general theorems which can be found in [21]. Some technical details, for instance, the construction of a Khasminskiı function, will be more complicated. Some models of symbiosis with other stochastic perturbations, for example $X^{2}(t)$ (see [8]), are also considered. 
2. Mathematical results and their interpretation. In this section we formulate the main result of our paper. We will study the stochastic model described by (2), (3) assuming that $b_{1} b_{2}<c_{1} c_{2}$. This is a natural assumption, because in the deterministic model (1) we observe that if $b_{1} b_{2} \geq c_{1} c_{2}$ then the sizes of both populations go to infinity. In other words, in the deterministic case too much symbiosis causes an unlimited growth of populations. In the stochastic model we can observe similar effects. Moreover, we will assume that the random noise for both populations is proportional to the number of individuals and is weakly correlated, i.e. $\rho_{11} \rho_{22}-\rho_{12} \rho_{21} \neq 0$. The asymptotic behaviour of system (2), (3) depends on the constants $b_{1}, b_{2}, c_{1}, c_{2}, \rho_{1}=$ $\sqrt{\rho_{11}^{2}+\rho_{12}^{2}}, \rho_{2}=\sqrt{\rho_{21}^{2}+\rho_{22}^{2}}, \tilde{a}_{1}=a_{1}-\rho_{1}^{2} / 2, \tilde{a}_{2}=a_{2}-\rho_{2}^{2} / 2$.

THEOREM 1. Let $b_{1} b_{2}<c_{1} c_{2}$. If $(X(t), Y(t))$ is a solution of system (2), (3), then for every $t>0$ the distribution of $(X(t), Y(t))$ has a density $U(t, x, y)$.

(I) If $\tilde{a}_{1}>0$ and $\tilde{a}_{2}>0$ then there exists a unique invariant density $U_{*}(x, y)$ such that

$$
\lim _{t \rightarrow \infty} \iint_{\mathbb{R}_{+}^{2}}\left|U(x, y, t)-U_{*}(x, y)\right| d x d y=0 .
$$

(II) If $\tilde{a}_{1}<0$ and $\tilde{a}_{2}<0$ then

$$
\lim _{t \rightarrow \infty} X(t)=0 \text { a.e. and } \lim _{t \rightarrow \infty} Y(t)=0 \text { a.e. }
$$

(III) If $\tilde{a}_{1}>0, \tilde{a}_{2}<0$ and $\tilde{a}_{1} b_{2}+\tilde{a}_{2} c_{1}>0$ then there exists a unique invariant density $U_{*}(x, y)$ such that

$$
\lim _{t \rightarrow \infty} \iint_{\mathbb{R}_{+}^{2}}\left|U(x, y, t)-U_{*}(x, y)\right| d x d y=0 .
$$

(IV) If $\tilde{a}_{1}>0, \tilde{a}_{2}<0$ and $\tilde{a}_{1} b_{2}+\tilde{a}_{2} c_{1}<0$ then $\lim _{t \rightarrow \infty} Y(t)=0$ a.e. and the distribution of the process $X(t)$ converges weakly to the measure which has the density $f_{*}(x)=C x^{2 \tilde{a}_{1} / \rho_{1}^{2}-1} e^{-2 c_{1} x / \rho_{1}^{2}}$, where $C=\left(2 c_{1} / \rho_{1}^{2}\right)^{2 \tilde{a}_{1} / \rho_{1}^{2}} / \Gamma\left(2 \tilde{a}_{1} / \rho_{1}^{2}\right)$.

REMARK 1 . In every case the support of the invariant density $U_{*}$ is $\mathbb{R}_{+}^{2}$. By the support of a measurable function $f$ we simply mean the set

$$
\operatorname{supp} f=\{(x, y) \in X: f(x, y) \neq 0\} .
$$

REMARK 2. In the statement of Theorem 1 we omit some cases. For example, we do not take into account the case in which $\tilde{a}_{1}<0, \tilde{a}_{2}>0$, $\tilde{a}_{2} b_{1}+\tilde{a}_{1} c_{2}>0$, because it is symmetrical to (III), nor the case when $\tilde{a}_{1}<0$, $\tilde{a}_{2}>0, \tilde{a}_{2} b_{1}+\tilde{a}_{1} c_{2}<0$ which is symmetrical to (IV).

REMARK 3. Theorem 1 has an interesting biological interpretation. The inequality $b_{1} b_{2}<c_{1} c_{2}$ means that the influence of symbiosis is smaller than 
the suppression of growth connected with the population size. This condition guarantees that the population cannot go to infinity in finite time and cannot die out in finite time. From (I) it follows that if stochastic perturbations are small for both populations then the sizes of the populations stabilize. However, if the stochastic perturbation is large for one population then this population can die out. It turns out that if the stochastic perturbation is large for one population and small for the second population but symbiosis coefficients are large then both populations survive. The main difference between the deterministic and stochastic models is that a large stochastic perturbation can cause the extinction of a population.

3. Existence and uniqueness of solution. In this section we prove that system (2), (3) has a unique and positive global (i.e. with no explosion in finite time) solution for any given initial value. In the proof we use the idea developed in [10], [8], [19]. First we introduce some notation. Let

$$
\mathbb{R}_{+}^{2}=\left\{\left(x_{1}, x_{2}\right) \in \mathbb{R}^{2}: x_{i}>0 \text { for all } i=1,2\right\} .
$$

We will study system (2), (3) with the initial condition $(X(0), Y(0)) \in \mathbb{R}_{+}^{2}$. We have the following result.

THEOREM 2. If $b_{1} b_{2}<c_{1} c_{2}$ then for any initial condition $(X(0), Y(0))$ $\in \mathbb{R}_{+}^{2}$ there is a unique solution $(X(t), Y(t))$ of system (2), (3) for $t \geq 0$ and the solution remains in $\mathbb{R}_{+}^{2}$ with probability 1 , that is, $(X(t), Y(t)) \in \mathbb{R}_{+}^{2}$ for all $t \geq 0$ almost surely.

Proof. Since the coefficients of equations (2), (3) are locally Lipschitz continuous, for any given initial condition $(X(0), Y(0)) \in \mathbb{R}_{+}^{2}$ there is a unique local solution $(X(t), Y(t))$ for $t \in\left[0, \tau_{e}\right)$, where $\tau_{e}$ is the explosion time. In order to show that this solution is global, we will show that $\tau_{e}=\infty$ a.e. Let $k_{0}>0$ be sufficiently large such that

$$
(X(0), Y(0)) \in\left[1 / k_{0}, k_{0}\right]^{2} .
$$

For each integer $k>k_{0}$ we define the stopping time

$$
\tau_{k}=\inf \left\{t \in\left[0, \tau_{e}\right):(X(t), Y(t)) \notin[1 / k, k]^{2}\right\}
$$

and we set $\inf \emptyset=\infty$. Clearly $\left(\tau_{k}\right)$ is an increasing sequence. Set $\tau_{\infty}=$ $\lim _{k \rightarrow \infty} \tau_{k}$, whence $\tau_{\infty} \leq \tau_{e}$ a.s. If we can show that $\tau_{\infty}=\infty$ a.s. then $\tau_{e}=\infty$ a.s. and consequently $(X(t), Y(t)) \in \mathbb{R}_{+}^{2}$ for all $t \geq 0$ a.s. In other words, to complete the proof we need to show that $\tau_{\infty}=\infty$ a.s. If this statement is false, then there is a pair of constants $T>0$ and $\varepsilon \in(0,1)$ such that

$$
P\left\{\omega \in \Omega: \tau_{\infty}(\omega) \leq T\right\}>\varepsilon .
$$

Consequently, there exists an integer $k_{1} \geq k_{0}$ such that

$$
P\left\{\omega \in \Omega: \tau_{k}(\omega) \leq T\right\} \geq \varepsilon \quad \text { for all } k \geq k_{1} .
$$


Define a $C^{2}$-function $V: \mathbb{R}_{+}^{2} \rightarrow \mathbb{R}$ by

$$
V(x, y)=b_{2} x_{0} F\left(x / x_{0}\right)+b_{1} y_{0} F\left(y / y_{0}\right),
$$

where $F(x)=x-\ln x-1$ and

$$
x_{0}=\frac{a_{2} b_{1}+a_{1} c_{2}}{c_{1} c_{2}-b_{1} b_{2}}, \quad y_{0}=\frac{a_{1} b_{2}+a_{2} c_{1}}{c_{1} c_{2}-b_{1} b_{2}} .
$$

If $(X(t), Y(t)) \in \mathbb{R}_{+}^{2}$, the Itô formula shows that

$$
\begin{aligned}
d V(X(t), Y(t))= & {\left[b_{2}\left(a_{1}+b_{1} Y(t)-c_{1} X(t)\right)\left(X(t)-x_{0}\right)\right.} \\
& +b_{1}\left(a_{2}+b_{2} X(t)-c_{2} Y(t)\right)\left(Y(t)-y_{0}\right) \\
& \left.+\frac{1}{2} \rho_{1}^{2} b_{2} x_{0}+\frac{1}{2} \rho_{2}^{2} b_{1} y_{0}\right] d t \\
& +b_{2}\left(X(t)-x_{0}\right) \rho_{11} d W_{1}(t)+b_{2}\left(X(t)-x_{0}\right) \rho_{12} d W_{2}(t) \\
& +b_{1}\left(Y(t)-y_{0}\right) \rho_{21} d W_{1}(t)+b_{1}\left(Y(t)-y_{0}\right) \rho_{22} d W_{2}(t) .
\end{aligned}
$$

Since $b_{1} b_{2}<c_{1} c_{2}$, we have

$b_{2}\left(a_{1}+b_{1} Y(t)-c_{1} X(t)\right)\left(X(t)-x_{0}\right)+b_{1}\left(a_{2}+b_{2} X(t)-c_{2} Y(t)\right)\left(Y(t)-y_{0}\right) \leq 0$.

We therefore obtain

$$
\begin{aligned}
E V\left(X\left(\tau_{k} \wedge T\right), Y\left(\tau_{k} \wedge T\right)\right) & \leq V(X(0), Y(0))+\alpha E\left(\tau_{k} \wedge T\right) \\
& \leq V(X(0), Y(0))+\alpha T,
\end{aligned}
$$

where $\alpha=\frac{1}{2} \rho_{1}^{2} b_{2} x_{0}+\frac{1}{2} \rho_{2}^{2} b_{1} y_{0}$. Set $\Omega_{k}=\left\{\omega \in \Omega: \tau_{k}(\omega) \leq T\right\}$ for $k \geq k_{1}$. Then $P\left(\Omega_{k}\right) \geq \varepsilon$. Note that for every $\omega \in \Omega_{k}$ there is some component of $\left(X\left(\tau_{k}(\omega)\right), Y\left(\tau_{k}(\omega)\right)\right.$ which equals either $k$ or $1 / k$, and hence by $(9)$,

or

$$
V\left(X\left(\tau_{k}(\omega)\right), Y\left(\tau_{k}(\omega)\right)\right) \geq b_{2} x_{0}\left(\frac{k}{x_{0}}-\ln \frac{k}{x_{0}}-1\right)
$$

or

$$
V\left(X\left(\tau_{k}(\omega)\right), Y\left(\tau_{k}(\omega)\right)\right) \geq b_{1} y_{0}\left(\frac{k}{y_{0}}-\ln \frac{k}{y_{0}}-1\right)
$$

or

$$
V\left(X\left(\tau_{k}(\omega)\right), Y\left(\tau_{k}(\omega)\right)\right) \geq b_{2} x_{0}\left(\frac{1}{k x_{0}}-\ln \frac{1}{k x_{0}}-1\right)
$$

$$
V\left(X\left(\tau_{k}(\omega)\right), Y\left(\tau_{k}(\omega)\right)\right) \geq b_{1} y_{0}\left(\frac{1}{k y_{0}}-\ln \frac{1}{k y_{0}}-1\right) .
$$

Let $C(k)$ be the minimum of the four right hand sides above. Then we have $\lim _{k \rightarrow \infty} C(k)=\infty$. From 10 ,

$$
V(X(0), Y(0))+\alpha T \geq E\left(1_{\Omega_{k}} V\left(X\left(\tau_{k}(\omega)\right), Y\left(\tau_{k}(\omega)\right)\right) \geq \varepsilon C(k),\right.
$$

where $1_{\Omega_{k}}$ is the indicator function of $\Omega_{k}$. Letting $k \rightarrow \infty$ leads to the contradiction

$$
\infty>V(X(0), Y(0))+\alpha T=\infty,
$$

so we must have $\tau_{\infty}=\infty$ a.s. 
4. Markov semigroups. Now we recall some definitions and theorems concerning Markov semigroups.

Let $(X, \Sigma, m)$ be a $\sigma$-finite measure space. Denote by $D$ the subset of the space $L^{1}=L^{1}(X, \Sigma, m)$ consisting of all densities, i.e.

$$
D=\left\{f \in L^{1}: f \geq 0,\|f\|=1\right\} .
$$

A linear mapping $P: L^{1} \rightarrow L^{1}$ is called a Markov operator if $P(D) \subset D$.

The Markov operator $P$ is called an integral operator if there exists a measurable function $k: X \times X \rightarrow[0, \infty)$ such that

$$
P f(x)=\int_{X} k(x, y) f(y) m(d y)
$$

for every density $f$. The function $k$ is called a kernel of the operator $P$. One can check that from the condition $P(D) \subset D$ it follows that

$$
\int_{X} k(x, y) m(d x)=1
$$

for almost all $y \in X$.

A family $\{P(t)\}_{t \geq 0}$ of Markov operators which satisfies these conditions:

(a) $P(0)=\mathrm{Id}$,

(b) $P(t+s)=P(t) P(s)$ for $s, t \geq 0$,

(c) for each $f \in L^{1}$ the function $t \mapsto P(t) f$ is continuous with respect to the $L^{1}$ norm,

is called a Markov semigroup. A Markov semigroup $\{P(t)\}_{t \geq 0}$ is called integral if for each $t>0$, the operator $P(t)$ is an integral Markov operator.

We also need two definitions concerning the asymptotic behaviour of a Markov semigroup. A density $f_{*}$ is called invariant if $P(t) f_{*}=f_{*}$ for each $t>0$. The Markov semigroup $\{P(t)\}_{t \geq 0}$ is called asymptotically stable if there is an invariant density $f_{*}$ such that

$$
\lim _{t \rightarrow \infty}\left\|P(t) f-f_{*}\right\|=0 \quad \text { for } f \in D .
$$

A Markov semigroup $\{P(t)\}_{t \geq 0}$ is called sweeping with respect to a set $A \in \Sigma$ if for every $f \in D$,

$$
\lim _{t \rightarrow \infty} \int_{A} P(t) f(x) m(d x)=0 .
$$

Theorem $3([13])$. Let $\{P(t)\}_{t \geq 0}$ be an integral Markov semigroup. Assume that the semigroup $\{P(t)\}_{t \geq 0}$ has only one invariant density $f_{*}$. If $f_{*}>0$ a.e. then the semigroup $\{P(t)\}_{t \geq 0}$ is asymptotically stable. In particular, if the integral Markov semigroup $\{P(t)\}_{t \geq 0}$ has a positive kernel and an invariant density then $\{P(t)\}_{t \geq 0}$ is asymptotically stable.

From Theorem 3 we obtain the following result. 
Corollary 1 ([16]). Let $X$ be a metric space and $\Sigma$ be the $\sigma$-algebra of Borel sets. Let $\{P(t)\}_{t \geq 0}$ be an integral Markov semigroup with a continuous kernel $k(t, x, y)$ for $t>0$, which satisfies (12) for all $y \in X$. Assume that for every $f \in D$,

$$
\int_{0}^{\infty} P(t) f d t>0 \quad \text { a.e. }
$$

Then the semigroup is asymptotically stable or sweeping with respect to compact sets.

A Markov semigroup $\{P(t)\}_{t \geq 0}$ that is asymptotically stable or sweeping from a sufficiently large family of sets (e.g. from all compact sets) is said to satisfy the Foguel alternative.

If we know that the semigroup satisfies the Foguel alternative we can exclude sweeping by showing that there exists a Khasminskiu function, defined as follows. Let $\mathcal{A}$ be the infinitesimal generator of a Markov semigroup $\{P(t)\}_{t \geq 0}$. Let $\mathcal{R}=(I-\mathcal{A})^{-1}$ be the resolvent operator at point 1 . Let $V: X \rightarrow[0, \infty)$ be a measurable function. Set

$$
D_{V}=\left\{f \in D: \int_{X} f(x) V(x) m(d x)<\infty\right\} .
$$

Then $V$ is called a Khasminskiu function for the Markov semigroup $\{P(t)\}_{t \geq 0}$ and a set $Z \in \Sigma$ if there exist $M>0$ and $\varepsilon>0$ such that

$$
\int_{X} V(x) \mathcal{R} f(x) d m(x) \leq \int_{X}(V(x)-\varepsilon) f(x) d m(x)+\int_{Z} M \mathcal{R} f(x) d m(x)
$$

for every $f \in D_{V}$. It is difficult to find a Khasminskiu function using the definition. In our case we can apply the following theorem.

TheOREM $4([12])$. Let $K$ be a compact set in $\mathbb{R}^{2}$. Assume that there exists a $C^{2}$-function $V: \mathbb{R}^{2} \rightarrow[0, \infty)$ such that

$$
\sup _{x \notin K} \mathcal{A}^{*} V(x)<0 .
$$

Then $V$ is a Khasminskiน function for the Markov semigroup $\{P(t)\}_{t \geq 0}$ and the set $K$.

5. Properties of trajectories. In order to investigate the properties of the solution of system (2), (3) we substitute $X(t)=e^{\xi(t)}, Y(t)=e^{\eta(t)}$. Then by the Itô formula we obtain

$$
\begin{aligned}
& d \xi(t)=\left(\tilde{a}_{1}+b_{1} e^{\eta(t)}-c_{1} e^{\xi(t)}\right) d t+\rho_{11} d W_{1}(t)+\rho_{12} d W_{2}(t), \\
& d \eta(t)=\left(\tilde{a}_{2}+b_{2} e^{\xi(t)}-c_{2} e^{\eta(t)}\right) d t+\rho_{21} d W_{1}(t)+\rho_{22} d W_{2}(t) .
\end{aligned}
$$

In this section we prove parts (II) and (IV) of Theorem 1. We will use the following property of solutions of a one-dimensional stochastic equation. 
Consider the stochastic equation

$$
d X(t)=\sigma(X(t)) d W(t)+b(X(t)) d t .
$$

Let

$$
s(x)=\int_{0}^{x} \exp \left\{-\int_{0}^{y} \frac{2 b(r)}{\sigma^{2}(r)} d r\right\} d y .
$$

If $s(-\infty)>-\infty$ and $s(\infty)=\infty$ then $\lim _{t \rightarrow \infty} X(t)=-\infty$ a.e.

The proof of parts (II) and (IV) of Theorem 1 is divided into the following lemmas.

Lemma 1. Let $(\xi(t), \eta(t))$ be a solution of system (15), (16). Assume that $\rho_{1} \neq 0$ and $\rho_{2} \neq 0$. If $b_{1} b_{2}<c_{1} c_{2}, \tilde{a}_{1}<0$ and $\tilde{a}_{2}<0$ then

$$
\lim _{t \rightarrow \infty} \xi(t)=-\infty \quad \text { and } \quad \lim _{t \rightarrow \infty} \eta(t)=-\infty \text { a.e. }
$$

Proof. Let

$$
\widetilde{W}(t)=\frac{\rho_{11}}{\rho_{1}} W_{1}(t)+\frac{\rho_{12}}{\rho_{1}} W_{2}(t), \quad \bar{W}(t)=\frac{\rho_{21}}{\rho_{2}} W_{1}(t)+\frac{\rho_{22}}{\rho_{2}} W_{2}(t) .
$$

Then $\widetilde{W}(t), \bar{W}(t)$ are two standard Wiener processes. We define

$$
W(t)=\frac{c_{2} \rho_{11}+b_{1} \rho_{21}}{\rho} W_{1}(t)+\frac{c_{2} \rho_{12}+b_{1} \rho_{22}}{\rho} W_{2}(t),
$$

where

$$
\rho=\sqrt{\left(c_{2} \rho_{11}+b_{1} \rho_{21}\right)^{2}+\left(c_{2} \rho_{12}+b_{1} \rho_{22}\right)^{2}} .
$$

Then $W(t)$ is also a standard Wiener process. Multiplying (15) by $c_{2}$ and 16 . by $b_{1}$ and adding the two equations we have

$$
d\left(c_{2} \xi(t)+b_{1} \eta(t)\right)=\left(\tilde{a}_{1} c_{2}+\tilde{a}_{2} b_{1}+\left(b_{1} b_{2}-c_{1} c_{2}\right) e^{\xi(t)}\right) d t+\rho d W(t) .
$$

Since $b_{1} b_{2}<c_{1} c_{2}$, from the comparison theorem ([4, Lemma 4, p. 120]) we get

$$
d\left(c_{2} \xi(t)+b_{1} \eta(t)\right) \leq\left(\tilde{a}_{1} c_{2}+\tilde{a}_{2} b_{1}\right) d t+\rho d W(t) .
$$

Consequently,

$$
\lim _{t \rightarrow \infty}\left(c_{2} \xi(t)+b_{1} \eta(t)\right)=-\infty \quad \text { a.e. }
$$

Thus for arbitrarily small $\varepsilon>0$ there exist $t_{0}$ and a set $\Omega_{\varepsilon}$ such that $\operatorname{Prob}\left(\Omega_{\varepsilon}\right)>1-\varepsilon$ and $\xi(t)<-\frac{b_{1}}{c_{2}} \eta(t)$ for $t \geq t_{0}$ and $\omega \in \Omega_{\varepsilon}$. It follows that

$$
d \eta(t) \leq\left(\tilde{a}_{2}+b_{2} e^{-\frac{b_{1}}{c_{2}} \eta(t)}\right) d t+\rho_{2} d \bar{W}(t)
$$

Consider the equation

$$
d \bar{\eta}(t)=\left(\tilde{a}_{2} / 2+b_{2} e^{-\frac{b_{1}}{c_{2}} \bar{\eta}(t)}\right) d t+\rho_{2} d \bar{W}(t) .
$$


The Fokker-Planck equation corresponding to 18 has a stationary density

$$
f_{*}(x)=C \exp \frac{2}{\rho_{2}^{2}}\left(\frac{\tilde{a}_{2}}{2} x-\frac{b_{2} c_{2}}{b_{1}} e^{-\frac{b_{1}}{c_{2}} x}\right),
$$

where $C$ is some constant. From the ergodic theorem ([4, Theorem 2, p. 141]) it follows that

$$
\lim _{t \rightarrow \infty} \frac{1}{t} \int_{0}^{t} e^{-\frac{b_{1}}{c_{2}} \bar{\eta}(s)} d s=\int_{-\infty}^{\infty} f_{*}(x) e^{-\frac{b_{1}}{c_{2}} x} d x .
$$

Since $f_{*}^{\prime}(x)=2 / \rho_{2}^{2}\left(\tilde{a}_{2} / 2+b_{2} e^{-\frac{b_{1}}{c_{2}} x}\right) f_{*}(x)$, we have

$$
\int_{-\infty}^{\infty} f_{*}(x) e^{-\frac{b_{1}}{c_{2}} x} d x=-\frac{\tilde{a}_{2}}{2 b_{2}}>0
$$

From 19, 20) we obtain

$$
\lim _{t \rightarrow \infty} \frac{1}{t} \int_{0}^{t} e^{-\frac{b_{1}}{c_{2}} \bar{\eta}(s)} d s=-\frac{\tilde{a}_{2}}{2 b_{2}} .
$$

In view of (17) we have

$$
\eta(t) \leq \bar{\eta}(t)+\frac{\tilde{a}_{2}}{2} t+C_{1}
$$

where $\bar{\eta}(t)$ is a solution of equation (18) and $C_{1}$ is some constant. Therefore

$$
\eta(t) \leq \tilde{a}_{2} t+b_{2} \int_{0}^{t} e^{-\frac{b_{1}}{c_{2}} \bar{\eta}(s)} d s+\rho_{2} \bar{W}(t)+C_{1} .
$$

From this and (21), $\lim _{t \rightarrow \infty} \lim \bar{W}(t) / t=0$, we obtain

$$
\limsup _{t \rightarrow \infty} \frac{\eta(t)}{t} \leq \frac{\tilde{a}_{2}}{2}<0
$$

Consequently, $\lim _{t \rightarrow \infty} \eta(t)=-\infty$ a.e. Using the symmetry of system (15), (16) we analogously show that $\lim _{t \rightarrow \infty} \xi(t)=-\infty$ a.e.

Lemma 2. Let $(\xi(t), \eta(t))$ be a solution of system (15), (16). Assume that $\rho_{1} \neq 0$ and $\rho_{2} \neq 0$. If $b_{1} b_{2}<c_{1} c_{2}, \tilde{a}_{1}>0, \tilde{a}_{2}<0$ and $\tilde{a}_{1} b_{2}+\tilde{a}_{2} c_{1}<0$ then $\lim _{t \rightarrow \infty} \eta(t)=-\infty$ a.e and the distribution of the process $\xi(t)$ converges weakly to a measure which has the density

$$
f_{*}(x)=C \exp \left(\frac{2}{\rho_{1}^{2}}\left(\tilde{a}_{1} x-c_{1} e^{x}\right)\right) .
$$

Proof. As in the proof of Lemma 1, we show that $\lim _{t \rightarrow \infty} \eta(t)=-\infty$ a.e. Thus for arbitrary $\varepsilon>0$ there exist $t_{0}$ and a set $\Omega_{\varepsilon}$ such that $\operatorname{Prob}\left(\Omega_{\varepsilon}\right)>$ $1-\varepsilon$ and $b_{1} e^{\eta(t, \omega)} \leq \varepsilon$ for $t \geq t_{0}$ and $\omega \in \Omega_{\varepsilon}$. Therefore

$$
\left(\tilde{a}_{1}-c_{1} e^{\xi(t)}\right) d t+\rho_{1} d \widetilde{W}(t) \leq d \xi(t) \leq\left(\tilde{a}_{1}+\varepsilon-c_{1} e^{\xi(t)}\right) d t+\rho_{1} d \widetilde{W}(t),
$$


where $\widetilde{W}(t)=\frac{\rho_{11}}{\rho_{1}} W_{1}(t)+\frac{\rho_{12}}{\rho_{1}} W_{2}(t)$. Let $\xi_{\varepsilon}(t)$ be a solution of the equation

$$
d \xi_{\varepsilon}(t)=\left(\tilde{a}_{1}+\varepsilon-c_{1} e^{\xi_{\varepsilon}(t)}\right) d t+\rho_{1} d \widetilde{W}(t)
$$

with the initial condition $\xi_{\varepsilon}(0)=\xi(0)$. Assume that $\xi_{0}(t)$ is a solution of the equation

$$
d \xi_{0}(t)=\left(\tilde{a}_{1}-c_{1} e^{\xi_{0}(t)}\right) d t+\rho_{1} d \widetilde{W}(t)
$$

with the initial condition $\xi_{0}(0)=\xi(0)$. Thus from the comparison theorem ([4, Lemma 4, p. 120]) it follows that $\xi_{0}(t) \leq \xi(t) \leq \xi_{\varepsilon}(t)$ a.e. Denote by $F_{\xi_{0}(t)}, F_{\xi(t)}, F_{\xi_{\varepsilon}(t)}$, respectively, the distributions of the processes $\xi_{0}(t)$, $\xi(t), \xi_{\varepsilon}(t)$. Then $F_{\xi_{0}(t)}(x) \geq F_{\xi(t)}(x) \geq F_{\xi_{\varepsilon}(t)}(x)$ for $x \in \mathbb{R}$. Every Markov semigroup connected with a diffusion process of a non-degenerate type is an integral semigroup which has a positive kernel. From Theorem 3 it follows that the densities of the process $\xi_{0}(t)$ converge in $L^{1}$ to an invariant density $f_{*}$ and the densities of the process $\xi_{\varepsilon}(t)$ converge in $L^{1}$ to an invariant density

$$
f_{\varepsilon}^{*}(x)=C \exp \left(\frac{2}{\rho_{1}^{2}}\left(\left(\tilde{a}_{1}+\varepsilon\right) x-c_{1} e^{x}\right)\right),
$$

where $C$ is some constant. In particular, $f_{*}=f_{0}^{*}$. Let

$$
F_{\varepsilon}^{*}(x)=\int_{-\infty}^{x} f_{\varepsilon}^{*}(s) d s
$$

Thus $F_{\xi_{\varepsilon}(t)}$ uniformly converges to $F_{\varepsilon}^{*}$ as $t \rightarrow \infty$ and $F_{\varepsilon}^{*}$ uniformly converges to $F_{0}^{*}$ as $\varepsilon \rightarrow 0$. Therefore the distribution of the process $\xi(t)$ converges weakly to the measure which has the density $f_{*}$.

6. Asymptotic stability. Let $(\xi(t), \eta(t))$ be a solution of 15, 16 such that the distribution of $(\xi(0), \eta(0))$ is absolutely continuous with density $v(x, y)$. Then the random variable $(\xi(t), \eta(t))$ has a density $u(x, y, t)$ and $u$ satisfies the Fokker-Planck equation:

$$
\frac{\partial u}{\partial t}=\frac{1}{2} \rho_{1}^{2} \frac{\partial^{2} u}{\partial x^{2}}+\left(\rho_{11} \rho_{21}+\rho_{12} \rho_{22}\right) \frac{\partial^{2} u}{\partial x \partial y}+\frac{1}{2} \rho_{2}^{2} \frac{\partial^{2} u}{\partial y^{2}}-\frac{\partial\left(f_{1} u\right)}{\partial x}-\frac{\partial\left(f_{2} u\right)}{\partial y},
$$

where $f_{1}(x, y)=\tilde{a}_{1}+b_{1} e^{y}-c_{1} e^{x}, f_{2}(x, y)=\tilde{a}_{2}+b_{2} e^{x}-c_{2} e^{y}$.

Now we introduce a Markov semigroup connected with the Fokker-Planck equation (22). Let $X=\mathbb{R}^{2}, \Sigma$ be the $\sigma$-algebra of Borel subsets of $X$, and $m$ be the Lebsegue measure on $(X, \Sigma)$. Let $P(t) v(x, y)=u(x, y, t)$ for $v \in D$. Since the operator $P(t)$ is a contraction on $D$, it can be extended to a contraction on $L^{1}\left(\mathbb{R}^{2}, \Sigma, m\right)$. Thus the operators $\{P(t)\}_{t \geq 0}$ form a Markov semigroup. Let $\mathcal{A}$ be the infinitesimal generator of the semigroup $\{P(t)\}_{t \geq 0}$, i.e.

$$
\mathcal{A} v=\frac{1}{2} \rho_{1}^{2} \frac{\partial^{2} v}{\partial x^{2}}+\left(\rho_{11} \rho_{21}+\rho_{12} \rho_{22}\right) \frac{\partial^{2} v}{\partial x \partial y}+\frac{1}{2} \rho_{2}^{2} \frac{\partial^{2} v}{\partial y^{2}}-\frac{\partial\left(f_{1} v\right)}{\partial x}-\frac{\partial\left(f_{2} v\right)}{\partial y} .
$$


The adjoint operator of $\mathcal{A}$ is of the form

$$
\mathcal{A}^{*} v=\frac{1}{2} \rho_{1}^{2} \frac{\partial^{2} v}{\partial x^{2}}+\left(\rho_{11} \rho_{21}+\rho_{12} \rho_{22}\right) \frac{\partial^{2} v}{\partial x \partial y}+\frac{1}{2} \rho_{2}^{2} \frac{\partial^{2} v}{\partial y^{2}}+f_{1} \frac{\partial v}{\partial x}+f_{2} \frac{\partial v}{\partial y} .
$$

By $\mathcal{P}(t, x, y, A)$ we denote the transition probability function for the diffusion process $(\xi(t), \eta(t))$, i.e. $\mathcal{P}(t, x, y, A)=\operatorname{Prob}((\xi(t), \eta(t)) \in A)$ and $(\xi(t), \eta(t))$ is a solution of 15 , 16) with the initial condition $(\xi(0), \eta(0))=$ $(x, y)$. Since $\rho_{11} \rho_{22}-\rho_{12} \rho_{21} \neq 0$, for each point $\left(x_{0}, y_{0}\right) \in \mathbb{R}^{2}$ and $t>0$ the measure $\mathcal{P}\left(t, x_{0}, y_{0}, \cdot\right)$ is absolutely continuous with respect to the Lebesgue measure. Denote by $k\left(t, x, y ; x_{0}, y_{0}\right)$ the density of $\mathcal{P}\left(t, x_{0}, y_{0}, \cdot\right)$. Thus

$$
P(t) v(x, y)=\int_{-\infty}^{\infty} \int_{-\infty}^{\infty} k(t, x, y ; \xi, \eta) v(\xi, \eta) d \xi d \eta
$$

and consequently $\{P(t)\}_{t \geq 0}$ is an integral Markov semigroup. The asymptotic stability of the semigroup $\{P(t)\}_{t \geq 0}$ implies the convergence in $L^{1}$ of the densities of the process $(\xi(t), \eta(t))$ to an invariant density. Therefore, instead of proving part (I) and (III) of Theorem 1, we show the asymptotic stability of the semigroup $\{P(t)\}_{t \geq 0}$. As $\rho_{11} \rho_{22}-\rho_{12} \rho_{21} \neq 0$, the semigroup $\{P(t)\}_{t \geq 0}$ connected with (22) has a continuous and positive kernel $k$. By Corollary 1 this semigroup satisfies the Foguel alternative. In order to exclude sweeping we construct a Khasminskil function. We have the following result.

Theorem 5. Assume that $b_{1} b_{2}<c_{1} c_{2}$. If $\tilde{a}_{1}>0, \tilde{a}_{2}>0$ or $\tilde{a}_{1}>0$, $\tilde{a}_{2}<0, \tilde{a}_{1} b_{2}+\tilde{a}_{2} c_{1}>0$ then the semigroup $\{P(t)\}_{t \geq 0}$ is asymptotically stable.

Proof. According to Theorem 4 we will construct a nonnegative $C^{2}$ function $V$ and a compact set $K \subset \mathbb{R}^{2}$ such that

$$
\sup _{x \notin K} \mathcal{A}^{*} V(x)<0 .
$$

Using similar arguments to those in [1] one can check that the existence of a Khasminskiı function implies that the semigroup is not sweeping from compact sets.

Let $\gamma_{1}=\log \left(c_{1} / b_{1}\right), \gamma_{2}=\log \left(b_{2} / c_{2}\right), \gamma=\left(\gamma_{1}+\gamma_{2}\right) / 2, \delta=\left(\gamma_{1}-\gamma_{2}\right) / 2$. As $b_{1} b_{2}<c_{1} c_{2}$ we have $\gamma_{2}<\gamma_{1}$ and $\delta>0$. If $d_{1}$ is a constant such that $0<d_{1}<\delta$ then we have the inequalities

$$
\gamma+d_{1}<\gamma_{1}, \quad \gamma-d_{1}>\gamma_{2} .
$$

First we consider the case when $\tilde{a}_{1}>0, \tilde{a}_{2}>0$. Let $a=\min \left(\tilde{a}_{1}, \tilde{a}_{2}\right)$ and

$$
d_{2}>\max \left\{\frac{6}{a}\left(\rho_{1}^{2}+\rho_{2}^{2}\right),-\left(\gamma+\gamma_{1}\right), \gamma+\gamma_{2}\right\} .
$$


We define

$$
\begin{aligned}
& D_{1}=\left\{(x, y) \in \mathbb{R}^{2}: x>z_{0}+d_{1}, y>z_{0}+\gamma+d_{1},\right. \\
& \left.x+\gamma-d_{1}<y<x+\gamma+d_{1}\right\}, \\
& D_{2}=\left\{(x, y) \in \mathbb{R}^{2}: x>z_{0}, y=x+\gamma+d_{1}\right\}, \\
& D_{3}=\left\{(x, y) \in \mathbb{R}^{2}: x>z_{0}+d_{1}, y=x+\gamma-d_{1}\right\} \text {, } \\
& D_{4}=\left\{(x, y) \in \mathbb{R}^{2}: x>z_{0}+d_{1}, y<-z_{0}-\gamma-d_{1}\right. \text {, } \\
& \left.-x-\gamma-d_{1}<y<-x-\gamma+d_{1}\right\}, \\
& D_{5}=\left\{(x, y) \in \mathbb{R}^{2}: x>z_{0}+d_{1}, y=-x-\gamma+d_{1}\right\} \text {, } \\
& D_{6}=\left\{(x, y) \in \mathbb{R}^{2}: x>z_{0}, y=-x-\gamma-d_{1}\right\} \text {, } \\
& D_{7}=\left\{(x, y) \in \mathbb{R}^{2}: x<-z_{0}-d_{1}, y<-z_{0}-\gamma-d_{1}\right. \text {, } \\
& \left.x-\gamma-d_{2}<y<x-\gamma+d_{2}\right\}, \\
& D_{8}=\left\{(x, y) \in \mathbb{R}^{2}: x<-z_{0}-d_{1}+d_{2}, y=x-\gamma-d_{2}\right\} \text {, } \\
& D_{9}=\left\{(x, y) \in \mathbb{R}^{2}: x<-z_{0}-d_{1}, y=x-\gamma+d_{2}\right\} \text {, } \\
& D_{10}=\left\{(x, y) \in \mathbb{R}^{2}: x<-z_{0}-d_{1}, y>z_{0}+\gamma+d_{1}\right. \text {, } \\
& \left.-x+\gamma-d_{1}<y<-x+\gamma+d_{1}\right\}, \\
& D_{11}=\left\{(x, y) \in \mathbb{R}^{2}: x<-z_{0}-d_{1}, y=-x+\gamma-d_{1}\right\} \text {, } \\
& D_{12}=\left\{(x, y) \in \mathbb{R}^{2}: x<-z_{0}, y=-x+\gamma+d_{1}\right\} \text {, } \\
& D_{13}=\left\{(x, y) \in \mathbb{R}^{2}: y>z_{0}+\gamma+d_{1}, y>x+\gamma+d_{1}, y>-x+\gamma+d_{1}\right\} \text {, } \\
& D_{14}=\left\{(x, y) \in \mathbb{R}^{2}: x>z_{0}+d_{1},-x-\gamma+d_{1}<y<x+\gamma-d_{1}\right\} \text {, } \\
& D_{15}=\left\{(x, y) \in \mathbb{R}^{2}: y<-z_{0}-\gamma-d_{1}, y<-x-\gamma-d_{1}, y<x-\gamma-d_{2}\right\} \text {, } \\
& D_{16}=\left\{(x, y) \in \mathbb{R}^{2}: x<-z_{0}-d_{1}, x-\gamma+d_{2}<y<-x+\gamma-d_{1}\right\} \text {, }
\end{aligned}
$$

where $z_{0}$ is a positive and sufficiently large constant. Next we define the function $z=V_{1}(x, y)$ for $z \geq z_{0}$ in the following way:

$$
\begin{gathered}
(x-z)^{4}+(y-(z+\gamma))^{4}=d_{1}^{4} \quad \text { for }(x, y) \in D_{1} \cup D_{2} \cup D_{3}, \\
(x-z)^{4}+(y+(z+\gamma))^{4}=d_{1}^{4} \quad \text { for }(x, y) \in D_{4} \cup D_{5} \cup D_{6}, \\
\left(x+z+d_{1}-d_{2}\right)^{4}+\left(y+z+d_{1}-d_{2}+\gamma\right)^{4}=d_{2}^{4} \quad \text { for }(x, y) \in D_{7} \cup D_{8} \cup D_{9}, \\
(x+z)^{4}+(y-(z+\gamma))^{4}=d_{1}^{4} \quad \text { for }(x, y) \in D_{10} \cup D_{11} \cup D_{12}, \\
z=y-\gamma-d_{1} \quad \text { for }(x, y) \in D_{13}, \quad z=x-d_{1} \quad \text { for }(x, y) \in D_{14}, \\
z=-y-\gamma-d_{1} \quad \text { for }(x, y) \in D_{15}, \quad z=-x-d_{1} \quad \text { for }(x, y) \in D_{16} .
\end{gathered}
$$

We show that there exist $\varepsilon>0$ and a compact set $K_{1} \subset \mathbb{R}^{2}$ such that $\mathcal{A}^{*} V_{1}(x, y) \leq-\varepsilon \quad$ for $(x, y) \notin K_{1}$.

Fix $0<\varepsilon<a / 2$. Let $K_{1}=\left[-z_{0}-d_{1}, z_{0}+d_{1}\right] \times\left[-z_{0}-\gamma-d_{1}, z_{0}+\gamma+d_{1}\right]$. 


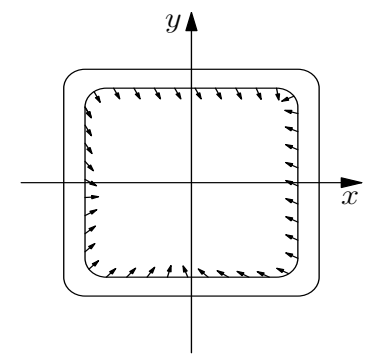

Fig. 1. The level curves of the Khasminskiu function if $b_{1} b_{2}<c_{1} c_{2}, \tilde{a}_{1}>0$ and $\tilde{a}_{2}>0$

We calculate

$$
\frac{\partial V_{1}}{\partial x}=\frac{(x-z)^{3}}{(x-z)^{3}+(y-(z+\gamma))^{3}}, \quad \frac{\partial V_{1}}{\partial y}=\frac{(y-(z+\gamma))^{3}}{(x-z)^{3}+(y-(z+\gamma))^{3}}
$$

and

$$
\frac{\partial V_{1}}{\partial x}+\frac{\partial V_{1}}{\partial y}=1, \quad \frac{\partial V_{1}}{\partial x}>0, \quad \frac{\partial V_{1}}{\partial y}>0
$$

for $(x, y) \in D_{1}$. Moreover,

$$
\frac{\partial^{2} V_{1}}{\partial x^{2}}=\frac{\partial^{2} V_{1}}{\partial y^{2}}=3(x-z)^{2}(y-(z+\gamma))^{2} \frac{(x-z)^{4}+(y-(z+\gamma))^{4}}{\left((x-z)^{3}+(y-(z+\gamma))^{3}\right)^{3}}
$$

and

$$
\frac{\partial^{2} V_{1}}{\partial x y}=-\frac{\partial^{2} V_{1}}{\partial x^{2}}<0
$$

for $(x, y) \in D_{1}$. Moreover,

$$
0<\frac{\partial^{2} V_{1}}{\partial x^{2}}=\frac{\partial^{2} V_{1}}{\partial y^{2}} \leq \frac{6}{d_{1}} .
$$

As $b_{1} e^{\gamma+d_{1}}-c_{1}<0, b_{2}-c_{2} e^{\gamma-d_{1}}<0$, for sufficiently large $z_{0}$ we obtain

and

$$
\begin{aligned}
\tilde{a}_{1}+b_{1} e^{y}-c_{1} e^{x} & <\tilde{a}_{1}+b_{1} e^{x+\gamma+d_{1}}-c_{1} e^{x}=\tilde{a}_{1}+e^{x}\left(b_{1} e^{\gamma+d_{1}}-c_{1}\right) \\
& <\tilde{a}_{1}+e^{z_{0}+d_{1}}\left(b_{1} e^{\gamma+d_{1}}-c_{1}\right)<-\frac{3}{d_{1}} \rho_{1}^{2}-\frac{3}{d_{1}} \rho_{2}^{2}-\varepsilon
\end{aligned}
$$

$$
\begin{aligned}
\tilde{a}_{2}+b_{2} e^{x}-c_{2} e^{y} & <\tilde{a}_{2}+b_{2} e^{x}-c_{2} e^{x+\gamma-d_{1}}=\tilde{a}_{2}+e^{x}\left(b_{2}-c_{2} e^{\gamma-d_{1}}\right) \\
& <\tilde{a}_{2}+e^{z_{0}+d_{1}}\left(b_{2}-c_{2} e^{\gamma-d_{1}}\right)<-\frac{3}{d_{1}} \rho_{1}^{2}-\frac{3}{d_{1}} \rho_{2}^{2}-\varepsilon
\end{aligned}
$$

From this and from 25, 26) we get

$$
\begin{gathered}
\mathcal{A}^{*} V_{1}(x, y) \leq \\
=\frac{3}{d_{1}} \rho_{1}^{2}+\frac{3}{d_{1}} \rho_{2}^{2}-\left(\frac{3}{d_{1}} \rho_{1}^{2}+\frac{3}{d_{1}} \rho_{2}^{2}+\varepsilon\right)\left(\frac{\partial V_{1}}{\partial x}+\frac{\partial V_{1}}{\partial y}\right) \\
=-\varepsilon
\end{gathered}
$$

for $(x, y) \in D_{1}$. Calculations in the other cases are similar or much easier and therefore we omit them. 
Now we consider the case when $\tilde{a}_{1}>0, \tilde{a}_{2}<0$ and $\tilde{a}_{1} b_{2}+\tilde{a}_{2} c_{1}>0$. Let $\mu=-2 \tilde{a}_{2} b_{2}, \nu=\tilde{a}_{1} b_{2}-\tilde{a}_{2} c_{1}$. It is easy to see that $\mu>0$ and $\nu>0$. Let $\alpha=\mu / \nu, y_{0}=-(3 \alpha / 2+1) z_{0}-\alpha d_{1}$. As before we define

$$
\begin{aligned}
& E_{1}= D_{1}, \quad E_{2}=D_{2}, \quad E_{3}=D_{3}, \\
& E_{4}=\left\{(x, y) \in \mathbb{R}^{2}: x>z_{0}+d_{1},\right. \\
&\left.y<y_{0},-x+z_{0}+y_{0}<y<-x+z_{0}+y_{0}+2 d_{1}\right\}, \\
& E_{5}=\left\{(x, y) \in \mathbb{R}^{2}: x>z_{0}+d_{1}, y=-x+z_{0}+y_{0}+2 d_{1}\right\}, \\
& E_{6}=\left\{(x, y) \in \mathbb{R}^{2}: x>z_{0}, y=-x+z_{0}+y_{0}\right\}, \\
& E_{7}=\left\{(x, y) \in \mathbb{R}^{2}: x<-z_{0}-d_{1}, y \leq-z_{0}\right\}, \\
& E_{8}=\left\{(x, y) \in \mathbb{R}^{2}:-z_{0}-d_{1} \leq x<z_{0} / 2, y<y_{0}\right\}, \\
& E_{9}=\left\{(x, y) \in \mathbb{R}^{2}: x \geq z_{0} / 2, y<y_{0}, y<-x+z_{0}+y_{0}\right\}, \\
& E_{10}=D_{10}, \quad E_{11}=D_{11}, \quad E_{12}=D_{12}, \quad E_{13}=D_{13}, \\
& E_{14}=\left\{(x, y) \in \mathbb{R}^{2}: x>z_{0}+d_{1},-x+z_{0}+y_{0}+2 d_{1}<y<x+\gamma-d_{1}\right\}, \\
& E_{15}=\left\{(x, y) \in \mathbb{R}^{2}: x<-z_{0}-d_{1},-z_{0}<y<-x+\gamma-d_{1}\right\} .
\end{aligned}
$$

Next we define the function $z=V_{2}(x, y)$ which is a modification of $V_{1}$. Let

$$
\begin{gathered}
V_{2}(x, y)=V_{1}(x, y) \quad \text { for }(x, y) \in E_{1} \cup E_{2} \cup E_{3} \cup E_{10} \cup E_{11} \cup E_{12} \cup E_{13}, \\
\begin{aligned}
&(x-z)^{4}+\left(y+\alpha z+(\alpha / 2+1) z_{0}+\right.\left.(\alpha-1) d_{1}\right)^{4} \\
&=d_{1}^{4} \quad \text { for }(x, y) \in E_{4} \cup E_{5} \cup E_{6}, \\
& \alpha x+y+\alpha z+z_{0}+\alpha d_{1}= 0 \quad \text { for }(x, y) \in E_{7} \cup E_{8}, \\
& 2 z+2 y+3 \alpha z_{0}+2 \alpha d_{1}=0 \text { for }(x, y) \in E_{9}, \\
& z=x-d_{1} \quad \text { for }(x, y) \in E_{14}, \quad z=-x-d_{1} \quad \text { for }(x, y) \in E_{15} .
\end{aligned}
\end{gathered}
$$

We show that there exist $\varepsilon>0$ and a compact set $K_{2} \subset \mathbb{R}^{2}$ such that

$$
\mathcal{A}^{*} V_{2}(x, y) \leq-\varepsilon \quad \text { for }(x, y) \notin K_{2} \text {. }
$$

Fix $0<\varepsilon<\min \left(\tilde{a}_{1}, \mu \tilde{a}_{1}+\nu \tilde{a}_{2}\right)$. Let $K_{2}=\left[-z_{0}-d_{1}, z_{0}+d_{1}\right] \times\left[y_{0}, z_{0}+\gamma\right.$ $\left.+d_{1}\right]$. Calculations are similar to the previous case. The main difference is in $E_{7} \cup E_{8}$. If $(x, y) \in E_{7} \cup E_{8}$ then

$$
\mathcal{A}^{*} V_{2}(x, y)=(1 / \mu)\left(-\mu \tilde{a}_{1}-\nu \tilde{a}_{2}+\left(\mu c_{1}-\nu b_{2}\right) e^{x}+\left(\nu c_{2}-\mu b_{1}\right) e^{y}\right) .
$$

As $\tilde{a}_{1} b_{2}+\tilde{a}_{2} c_{1}>0, b_{1} b_{2}<c_{1} c_{2}$ and $\tilde{a}_{2}<0$ thus $-\mu \tilde{a}_{1}-\nu \tilde{a}_{2}<0, \mu c_{1}-\nu b_{2}<0$ and

$$
\begin{aligned}
\nu c_{2}-\mu b_{1} & =b_{2}\left(\tilde{a}_{1} c_{2}+\tilde{a}_{2} b_{1}\right)+\tilde{a}_{2}\left(b_{1} b_{2}-c_{1} c_{2}\right) \\
& >\frac{b_{1} b_{2}}{c_{1}}\left(\tilde{a}_{1} b_{2}+\tilde{a}_{2} c_{1}\right)+\tilde{a}_{2}\left(b_{1} b_{2}-c_{1} c_{2}\right)>0 .
\end{aligned}
$$

Consequently, for $(x, y) \in E_{7}$ we have

$$
\mathcal{A}^{*} V_{2}(x, y) \leq(1 / \mu)\left(-\mu \tilde{a}_{1}-\nu \tilde{a}_{2}+\left(\nu c_{2}-\mu b_{1}\right) e^{-z_{0}}\right)<-\varepsilon,
$$




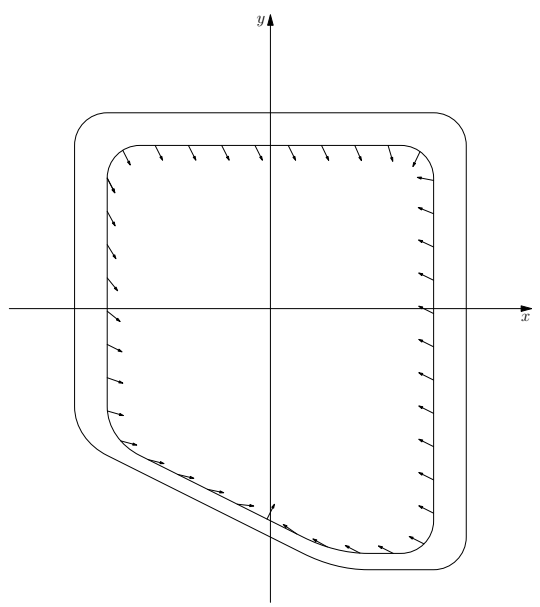

Fig. 2. The level curves of the Khasminskiu function if $b_{1} b_{2}<c_{1} c_{2}, \tilde{a}_{1}>0, \tilde{a}_{2}<0$, $\tilde{a}_{1} b_{2}+\tilde{a}_{2} c_{1}>0$

and for $(x, y) \in E_{8}$ we obtain

(30) $\mathcal{A}^{*} V_{2}(x, y) \leq(1 / \mu)\left(-\mu \tilde{a}_{1}-\nu \tilde{a}_{2}+\left(\nu c_{2}-\mu b_{1}\right) e^{-(3 \alpha / 2+1) z_{0}-\alpha d_{1}}\right)<-\varepsilon$,

because $z_{0}$ is sufficiently large.

\section{References}

[1] D. H. Boucher, The Biology of Mutualism, Oxford Univ. Press, 1988.

[2] S. Chessa and H. Fujita Yashima, The stochastic equation of predator-prey population dynamics, Boll. Un. Mat. Ital. Sez. B (8) 5 (2002), 789-804 (in Italian).

[3] G. F. Gause and A. A. Witt, Behaviour of mixed populations and the problem of natural selection, Amer. Naturalist 69 (1935), 596-609.

[4] I. I. Gikhman and A. V. Skorohod, Stochastic Differential Equations, Springer, Berlin, 1972.

[5] R. Z. Khasminskiǔ, Stochastic Stability of Differential Equations, Sijthoff \& Noordhoff, Alphen aan den Rijn, 1980.

[6] V. A. Kostitzin, Symbiose, parasitisme et évolution, Hermann, Paris, 1934.

[7] A. Lasota and M. C. Mackey, Chaos, Fractals and Noise. Stochastic Aspects of Dynamics, Springer Appl. Math. Sci. 97, Springer, New York, 1994.

[8] X. Mao, G. Marion and E. Renshaw, Environmental Brownian noise suppresses explosions in population dynamics, Stoch. Process. Appl. 97 (2002), 95-100.

[9] J. D. Murray, Mathematical Biology. I: An Introduction, Springer, New York, 2002.

[10] K. Narita, No explosion criteria for stochastic differential equations, J. Math. Soc. Japan 34 (1982), 191-203.

[11] K. Pichór and R. Rudnicki, Stability of Markov semigroups and applications to parabolic systems, J. Math. Anal. Appl. 215 (1997), 56-74.

[12] - - - Asymptotic behaviour of Markov semigroups and applications to transport equations, Bull. Polish Acad. Sci. Math. 45 (1997), 279-397. 
[13] K. Pichór and R. Rudnicki, Continuous Markov semigroups and stability of transport equations, J. Math. Anal. Appl. 249 (2000), 668-685.

[14] A. Rescigno and W. Richardson, The struggle for life I: Two species, Bull. Math. Biophys. 29 (1967), 377-388.

[15] R. Rudnicki, On asymptotic stability and sweeping for Markov operators, Bull. Polish Acad. Sci. Math. 43 (1995), 245-262.

[16] -, Long-time behaviour of stochastic prey-predator model, Stoch. Process. Appl. 108 (2003), 93-107.

[17] R. Rudnicki and K. Pichór, Influence of stochastic perturbation on prey-predator systems, Math. Biosci. 206 (2007), 108-119.

[18] K. Rudnicki, K. Pichór and M. Tyran-Kamińska, Markov semigroups and their applications, in: Dynamics of Dissipation, P. Garbaczewski and R. Olkiewicz (eds.), Lecture Notes in Phys. 597, Springer, Berlin, 215-238.

[19] E. Tornatore and S. M. Buccellato, On stochastic SIR model, Appl. Math. (Warsaw) 34 (2007), 389-400.

[20] E. Tornatore, L. Manca and H. Fujita Yashima, Comportamento asintotico della soluzione del sistema di equazioni stocastiche per due specie in competizione, Ist. Lombardo Accad. Sci. Lett. Rend. A 136-137 (2004), 151-183.

[21] F. Wu and S. Hu, Stochastic delay Kolmogorov-type population dynamics, J. Math. Anal. Appl. 347 (2008), 534-549.

Urszula Skwara

Institute of Mathematics

Maria Curie-Skłodowska University

Pl. M. Curie-Skłodowskiej 1

20-031 Lublin, Poland

E-mail: uskwara@umcs.lublin.pl, uskwara@o2.pl

Received 15.3.2009

and in final form 25.9.2009 